\title{
Risk Perceptions Related to SARS and Avian Influenza: Theoretical Foundations of Current Empirical Research
}

\author{
Anja Leppin • Arja R. Aro
}

Published online: 12 February 2009

(C) International Society of Behavioral Medicine 2009

\begin{abstract}
Background The outbreak of severe acute respiratory syndrome in 2003 and the subsequent emergence of the H5N1 virus have highlighted the threat of a global pandemic influenza outbreak. Planning effective public health control measures for such a case will be highly dependent on sound theory-based research on how people perceive the risks involved in such an event.

Purpose The present article aims to review theoretical models and concepts underlying current empirical research on pandemic influenza risk perception.

Method A review was conducted based on 28 empirical studies from 30 articles which were published between 2003 and 2007.

Results Concepts of risk perception mostly seemed more pragmatic than theory-based and were highly heterogeneous, for instance, in terms of conceptualizing risk perception as an exclusively cognitive or as a cognitive and emotional phenomenon or whether the concept was dominated by expectancy or expectancy and value components. Similarly, the majority of studies investigating risk perceptions and protective behaviors were not model-based. Conclusions The current body of knowledge can only provide preliminary insights. Unlike the reviewed studies, which were mostly launched as a rapid response to outbreak situations, future research will have to invest more strongly into theoretical work to provide sounder evidence.
\end{abstract}

Keywords Severe acute respiratory syndrome (SARS) . Avian influenza $\cdot$ Risk perception $\cdot$ Theory $\cdot$ Models

\footnotetext{
A. Leppin $(\bowtie) \cdot$ A. R. Aro

University of Southern Denmark, Institute of Public Health,

Unit for Health Promotion,

Niels Bohrs Vej 9,

6700 Esbjerg, Denmark

e-mail: aleppin@health.sdu.dk
}

\section{Introduction}

Newly emerging respiratory infectious diseases like severe acute respiratory syndrome (SARS) and avian influenza have created novel challenges to traditional nonmedical public health containment strategies [1]. In the initial absence of effective vaccination, measures such as quarantine, travel restrictions, and the promotion of individual protection behaviors such as frequent hand washing, wearing of face masks, or avoiding public gatherings were the measures of choice during the SARS outbreak and will be again should another pandemic influenza, caused, for instance, by H5N1, occur. The effectiveness of such control measures depends fundamentally on the public's willingness to cooperate, which again is likely to be associated with the level of personal risk people perceive. Sound empirical data about the ways lay persons perceive such risks are therefore essential to develop health communication strategies and successfully devise containment plans. Collecting such a sound database, however, requires first a thorough theoretical understanding of what constitutes lay persons' risk perceptions of pandemic influenza, how these perceptions emerge, as well as how they influence protective behaviors.

Therefore, as an addition to this special issue's original contributions on perceptions of SARS and avian influenza risk, the present article will take a more general perspective and explore the theoretical foundations of risk perception research within the specific context of pandemic influenza ${ }^{1}$. Specifically, the present article aims to review theoretical models and concepts underlying empirical research on

\footnotetext{
${ }^{1}$ The term pandemic influenza will be used as a generic term for lifethreatening respiratory infectious diseases with epidemic-pandemic potential, of which SARS and H5NI are only two examples, which might be succeeded by further outbreaks of other viruses.
} 
perceptions related to SARS and avian influenza as the two most recent threatening cases of respiratory virus outbreaks. The particular focus of this review will be on how risk perception is conceptualized and also on which models have been chosen by current empirical studies to test for the relationship between SARS or avian influenza risk perception and protective behaviors.

\section{Current Concepts of Risk Perception}

The Psychometric Paradigm and Expectancy-Value Models

Concepts of risk have been shaped in the light of different scientific traditions and paradigms. Psychological research on risk perception has mainly been dominated by the psychometric paradigm on the one hand and the subjective expected utility approach on the other [2]. The psychometric paradigm [3,4], wanting to investigate what characterizes risk in the public's view, has tried to map lay people's perception of different types of hazards in factorial space, describing the decisive dimensions as unknown risk and dread risk, with the latter dimension being the most important determinant of a "risk image." While unknown risk describes phenomena which are perceived of as new, nonobservable, unknown to science, and whose effects are delayed, dread risk has been defined by characteristics such as involuntariness, uncontrollability, fatal consequences, and catastrophic dimensions. Technological hazards, such as nuclear power or genetically modified food, tend to be perceived of as unknown risks and, just like natural disasters, often convey high dread potential. Lifestyle behaviors like smoking, unhealthy eating habits, or car speeding, on the other hand, are usually thought of as observable and well known and thus rank relatively low on the factor unknown risk. Also, lay persons tend to perceive these behaviors as voluntary, controllable, and as affecting people one at a time rather than simultaneously endangering whole populations. So, in the public's eye lifestyle factors lack the dread potential of technological risks and hence often seem more acceptable than the former.

While there is yet no empirical evidence for this, one might speculate that the specific characteristics of disease threats such as SARS or avian influenza would place them conceptually closer to technological risks or to natural disasters than to individual risk behaviors. Just as for technological risks, an initial lack of knowledge about the origin or the life course of a newly emerging virus and the fact that the infection process in itself is unobservable and that visible effects are delayed might produce high rankings on the factor unknown risk. In addition, pandemic influenza, just like technological and natural hazards, has catastrophic potential, as infection could be fatal, and large numbers of people would be affected at the same time within the same geographical region. Beyond this, global mobility is bound to further add to catastrophic impact by rapid spread of a respiratory virus to distant regions of the world. Also, as specific vaccines need time to be developed and as $100 \%$ effective medication is unlikely to be available for a considerable time span, these diseases are difficult to control - all aspects which suggest that pandemic influenza would rank high on the dread risk factor just like technological risks or natural disasters. On the other hand, it is particularly the issue of control which might make perceptions of pandemic influenza more ambiguous. Like in the case of natural disasters or technological hazards, there is an outside threat, in this case a virus, which might seem difficult to control. However - different from natural or technological hazards - this particular outside agent depends on everyday human behavior to spread, i.e., social contact and hygiene behaviors. Even more than for instance smoking, car speeding, or alcohol consumption, these behaviors are extremely common, well known, and observable, more or less voluntary and - at least theoreticallymodifiable or controllable. Objects with these characteristics are rarely perceived as "high risk" by lay persons, as research based on the psychometric paradigm has demonstrated [4]. Thus, at least during the initial phase of an outbreak, there might be a difference between perceived threat of the disease and the risk potential actually attributed to one's own individual contact behaviors.

This notion needs empirical testing, just as the assumption that the aggregate-level data of the psychometric paradigm are transferable to the individual level. If proven valid, however, this phenomenon might have consequences for planning public health control strategies. To combat disease spread in the initial phases of a pandemic influenza outbreak, where no vaccine is available, control measures will have to rely on influencing the extent (e.g., avoiding public gatherings, closing down schools and work places) and mode (e.g., wearing of face masks, no handshaking, frequent hand washing with soap, etc.) of human contact behaviors. Thus, within a very short time span, social interaction would have to transform from an integral, essential, mostly beneficial, or at least unquestioned part of daily life into risk-fraught, even potentially lethal behavior. How prepared or reluctant people are to make that mental shift, how effective risk perceptions are in initiating protective behaviors without creating panic and problematic social exclusion processes, and which factors influence these processes are key questions for planning containment and communication strategies.

Unlike the psychometric paradigm, which has been developed to describe and investigate aggregate-level characteristics of various types of hazards perceived of as "low risk" or "high risk," expectancy-value models have 
focused on how individual risk perception influences decision making and behavior. Based on the subjective expected utility (SEU) approach [2], which posited that people's choices are a product of assessments of probability and utility/desirability of options, health-related approaches such as the Health Belief Model (HBM) [5, 6] or Protection Motivation Theory (PMT) $[7,8]$ have defined risk in line with the technical understanding of risk, i.e., as the likelihood of contracting a disease multiplied by disease severity. Together with other types of expectancies, like outcome expectancies (or cost-benefit perceptions) and self-efficacy expectations, these perceptions of risk are then presumed to determine health-protective behaviors such as using health care services, refraining from smoking, or being physically active. These models have found widespread application as well as a considerable degree of empirical support (see below) and might therefore also provide a sound basis for answering questions on how far pandemic influenza risk perceptions can be relied upon as motivating factors for protective behaviors. Beyond a mere focus on risk perception, they can also help reveal how other types of cognition, such as outcome expectancies, about control measures as well as judgements on costs involved in such measures, such as cutting or reducing human contacts, might support or counteract the motivational processes initiated by risk perception.

\section{Risk as Cognition or Risk as Emotion?}

The Health Belief Model and Protection Motivation Theory, however, also have shortcomings which could limit their use for studying pandemic influenza risk perception. One common criticism of these models pertains to their strong focus on conscious-rationalist situational appraisal which falls short of capturing the emotional processes involved in human decision making. Dualprocess models have for a long time been suggesting that analytic-central and emotional-heuristic processes work in concert to select and prioritize decisions and behavioral reactions $[9,10]$, and under certain circumstances emotions may even be the dominant force. Damasio [11] has thus suggested that automated incentive or alarm signals linked to pleasant or unpleasant "gut feelings" often precede cognitive reasoning. In a similar vein, processing theories have emphasized the importance of an emotion-cognition pathway [12-14]. Based on these ideas, the "risk as feelings" or "risk as values" approach [15-17], just as the notion of an "affect heuristic" $[18,19]$, have posited that when making judgements individuals draw on two processes of evaluation, which reside in two distinct, if interrelated, systems of decision making. These are an analytic-rational system based on logical reasoning and probability judgements, on the one hand, and an experiential system, on the other, where direct automatic visceral reactions influence decision making, based on images, metaphors, and related emotions.

While the HBM or PMT, unlike the original SEU approach, do include an emotional component, i.e., fear, they represent a cognitive appraisal framework $[20,21]$ in assuming that it is mostly cognitions which give raise to emotions, i.e., cognitive risk assessment determines experience of fear. This framework might well apply for behaviors aimed at fending off long-term chronic disease such as screening participation or lifestyle behaviors. In such cases, fear is likely to be less imminent and therefore secondary to more rational reflections about gains and losses related to protective behavior. However, in an acute threat situation like an influenza pandemic, emotional aspects might gain more immediate importance. This is all the more likely if, during the early stages of an outbreak, experts are unable to make more than tentative statements and provide partly contradictory prognoses and recommendations as it happened with SARS. Under these circumstances cognitive risk assessments might be severely hampered by lack of evidence-based information. Consequently, individuals might have little choice but to rely on experiential judgement. However, what role exactly an affect- or emotionbased judgement plays in interaction with risk-related cognitions is still an issue in need of clarification.

\section{The Social Context of Risk Perception}

Another issue which warrants more attention than it has so far received in risk perception research is the social context of risk perception. Health behavior models such as the HBM or PMT have mainly focused on how risk perceptions and other cognitions influence behavior, while the question of how risk perceptions are formed in the first place has met with little attention. Public risk perception is thus mostly conceived of as an aggregate of purely individual assessments - a perspective which neglects cultural and subcultural frames of reference [22]. Slovic [4], in contrast, has emphasized the importance of worldviews, which can be perceived of as sociocultural attitudes guiding individual risk perception by providing normative orientation. Similarly, social representation theory [23] or the social amplification of risk framework [24, 25] have emphasized the importance of collective, (sub)cultural-social dynamics which shape individual ways to perceive of and react to risk.

Even more than other types of diseases, infections inherently involve a social dimension due to the social basis of transmission. Risk perception here pertains not only to the self but also to the likelihood of close others being affected. Beyond that, the possibility that oneself might become instrumental in infecting others can evoke 
anticipations of guilt and blame. Furthermore, anticipation of social exclusion processes affecting those who have become "cases" or those who are facing the frontline combating disease, like health care workers, are bound to become a part of pandemic influenza risk conception and therefore might also have an influence on risk-related behaviors. While such processes can be assumed to be relevant for all societies, it seems probable that such "social risk perceptions" are even stronger in the collectivist societies of Asia than in the more individualist cultures of the West. Factoring in such processes of perceiving and reacting to collective threat is unquestionably an essential element of pandemic influenza preparedness.

\section{Models of the Risk Perception-Behavior Relationship}

In line with the SEU approach, health behavior models assume that high perceived personal risk can be expected to motivate people into action. However, it has also been pointed out that not all empirical studies which have tested this assumption have yielded particularly large effects or have borne out this type of effect at all $[26,27]$. This heterogeneous state of empirical findings reflects conflicting underlying psychological processes, but also methodological problems involved in the respective studies.

In terms of psychological processes, one explanation for the diversity of findings is that very high risk perceptions in some population subgroups, instead of mobilizing people into action, might evoke feelings of helplessness which could paralyze rather than provoke protective behaviors. A threat like pandemic influenza, where in the initial absence of a vaccine the efficacy of countermeasures must remain unclear, might be particularly prone to such no-action responses. Vice versa, some people will downplay risk as a kind of coping strategy and thus report lower risk perception which, however, would not necessarily keep them from initiating protective action. Testing such alternative pathways requires the investigation not only of cognitive but also of emotional aspects of or reactions to risk perception.

Also, risk perceptions are not alone in determining protective behaviors. In the absence of positive outcome expectancies for protective behaviors or high self-efficacy, in terms of feeling able to perform these behaviors, high risk perceptions are unlikely to be particularly effective. Another important factor in this context is perceived social norms as outlined by the Theory of Reasoned Action/ Theory of Planned Behavior [28, 29]. Normative influences can explain why even those with low personal risk perceptions sometimes adopt protective behaviors. During an outbreak protective behaviors are widely recommended and visibly performed by a great number of people, so that their practice acquires normative status. During the SARS epidemic this might have happened with behaviors such as wearing of face masks, frequent hand washing, or avoidance of public gatherings, where the issue was not only to protect oneself but also to not be perceived as someone who puts others at risk.

Brown [30] has pointed out yet another reasoning process which might cancel out any protective motivational impulses of risk appraisal: Belief modification as a means to reduce cognitive dissonance. In a prospective study on car speeding the author found an inverse relationship between car speeding and perception of accident risk, which he accordingly interpreted as an attempt to reduce dissonance or else as a learning effect signaling that, in the absence of (immediate) negative consequences, risk perceptions might decrease [31]. For pandemic influenza such an effect could occur if people who escape infection during an initial wave get careless, based on the assumption that they could be "immune." Finding out whether such processes actually take place requires longitudinal process approaches and also in-depth explorations of people's ways of reasoning about their personal risk perceptions.

Finally, the impact of risk perceptions on behavior is likely to differ depending on the specificity of the risk assessments. Thus, perceived general risk of contracting SARS or avian influenza might relate less strongly to specific types of protective behavior such as not shaking hands, frequently washing hands, etc. than the more specific risks perceived as being involved in these particular behaviors.

From a methodological perspective, Brewer et al. [26] (see also [27]) have recently highlighted another series of problems. One of these is the failure to condition the risk question on not taking action [27]. Not accounting for anticipated or intended positive behavior change can lead to an underestimation of risk because it is likely that some people assess their risk as being low due to already factoring in a change towards anticipated or already accomplished protective behavior. The authors, however, also pointed out that the relevance of this issue depends on whether the precaution is actually expected to eliminate or at least reduce the risk (response efficacy). For investigating pandemic influenza, this type of validity threat might therefore be less imminent than, e.g., for a study on the relationship between perceived risk of "normal" influenza and getting a vaccination. Even so, such effects are unlikely to be nonexistent, so that perceived pandemic influenza risk research should also profit from assessments with and without (anticipated or actual) behavior change.

Interpretational ambiguity can, of course, also result from temporal relations between variables. In cross-sectional studies perceptions of risk for "ordinary influenza" in people who have already been vaccinated are bound to be 
affected by their expectation of being protected by the vaccination, hence their lowered risk perceptions - which then should not be interpreted as an indication of higher risk perception leading to less protective behavior. Brewer et al. [26] explicitly tried to disentangle the various types of effects by investigating different temporal hypotheses of risk perception about Lyme disease and protective behavior, i.e., getting vaccinated. Based on their findings, they once more emphasized the well-known predicament of crosssectional studies. Even though often claiming to test a behavior motivation hypothesis, studies based on correlational designs cannot perform more than a test of an accuracy hypothesis, stating that people who engage in more risk behaviors should also endorse higher risk perceptions. Testing a behavior motivation hypothesis on the other hand requires a longitudinal design, looking at whether higher risk perception at $\mathrm{t} 1$ leads to a stronger increase in protective behavior at $\mathrm{t} 2$. Yet another effect is suggested by the risk appraisal hypothesis, which suggests that risk appraisal will change as a result of behavior change, i.e., it will decrease once positive behavior change has been initiated. The two tests should thus generate inverse correlations. In the first phase, when the behavior motivation model is tested, perceived risk should be positively related to preventive behavior. In the second phase, however, when the risk appraisal model is under investigation, a negative relationship between preventive behavior and risk perception should be expected. Empirical findings of the Brewer et al. [26] study supported these assumptions, showing that higher initial risk judgements concerning Lyme disease encouraged people in favor of vaccination. Taking action subsequently reduced risk perception, indicating also that people who had been vaccinated viewed their risk as lower than those who had not, thereby confirming the accuracy hypothesis. The quite common failure to explicitly distinguish these processes contributes to misinterpretation of findings and misattribution of effects.

The long list of open questions and unsolved issues in risk perception research certainly sets an ambitious research agenda. The purpose of the present study is therefore, first, to take stock of the status quo with regard to the area of pandemic influenza risk, i.e., to investigate the theoretical basis of current empirical research on SARS and avian influenza risk perceptions. Relevant empirical studies will be explored in terms of (1) their conceptual and operational definitions of risk perception and (2) the theoretical models used for investigating the role of risk perceptions as determinants of protective behaviors. The review uses a descriptive-quantitative approach insofar as it not only tries to describe the different concepts and models of risk used in the various studies and also to quantify the amount of studies falling into different categories in order to give a more precise account of the state of theoretical groundwork behind current empirical risk perception research. What the paper does not provide is a review on empirical findings of the current risk perception research itself in terms of degree of risk perception in different populations, or strength of the risk perception-health behavior relationship.

\section{Method}

The analysis is based on 28 empirical studies from 30 publications $^{2}$ focusing on the threat posed by SARS and avian influenza and the subjective perceptions related to these health hazards (see Table 1). The search for studies published between January of 2003, the year of the SARS outbreak, and October 2007 was conducted with the help of different databases (PUBMED, EMBASE, PSYCLIT, and CINAHL), alternatively combining the term "risk perception" with "SARS," "avian flu," "avian influenza," and "pandemic influenza." Additionally, reference sections of the papers obtained were searched for further articles. A study was included only if it explicitly referred to "risk perception" or else to subcomponents of the perceived risk concept, i.e., "perceived susceptibility" or "perceived vulnerability." Thus, studies dealing exclusively with, for instance, SARS-related "distress" or "threat" without explicitly referring to the perceived risk concept were not included in the review.

\section{Categorization Procedures}

Exploring risk concepts was made difficult by the fact that the large majority of studies provided no explicit theoretical definition of how risk perception was conceived of. As a consequence, risk concepts had to be traced back to and deduced from the operational measures used. Descriptions of these assessments, however, were not always sufficiently comprehensive or particularly precise, and the terminology used in the introductory or discussion sections of the articles were not always consistent with the measures actually applied and/or compatible with conventional definitions, all of which contributed to the complexity of the classification procedure. This multifaceted picture was further complicated by the fact that many studies, besides explicitly introducing measures of risk perception, additionally assessed aspects which could be considered as risk-

\footnotetext{
${ }^{2}$ In two cases, publications clearly seemed to be based on the same study. These were therefore counted as one study each; however, in both cases, for the information in Table 1, all four articles were used.
} 


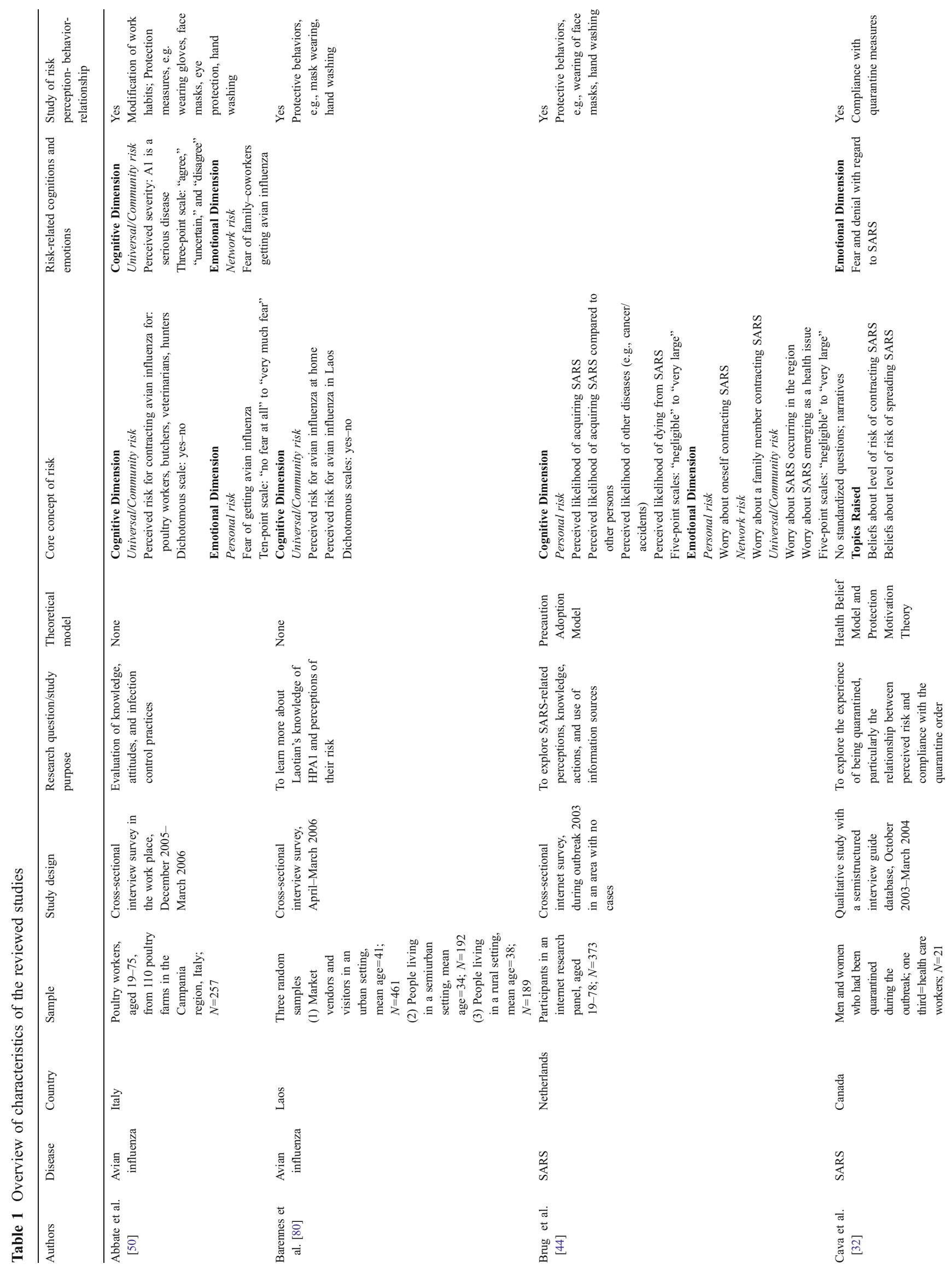



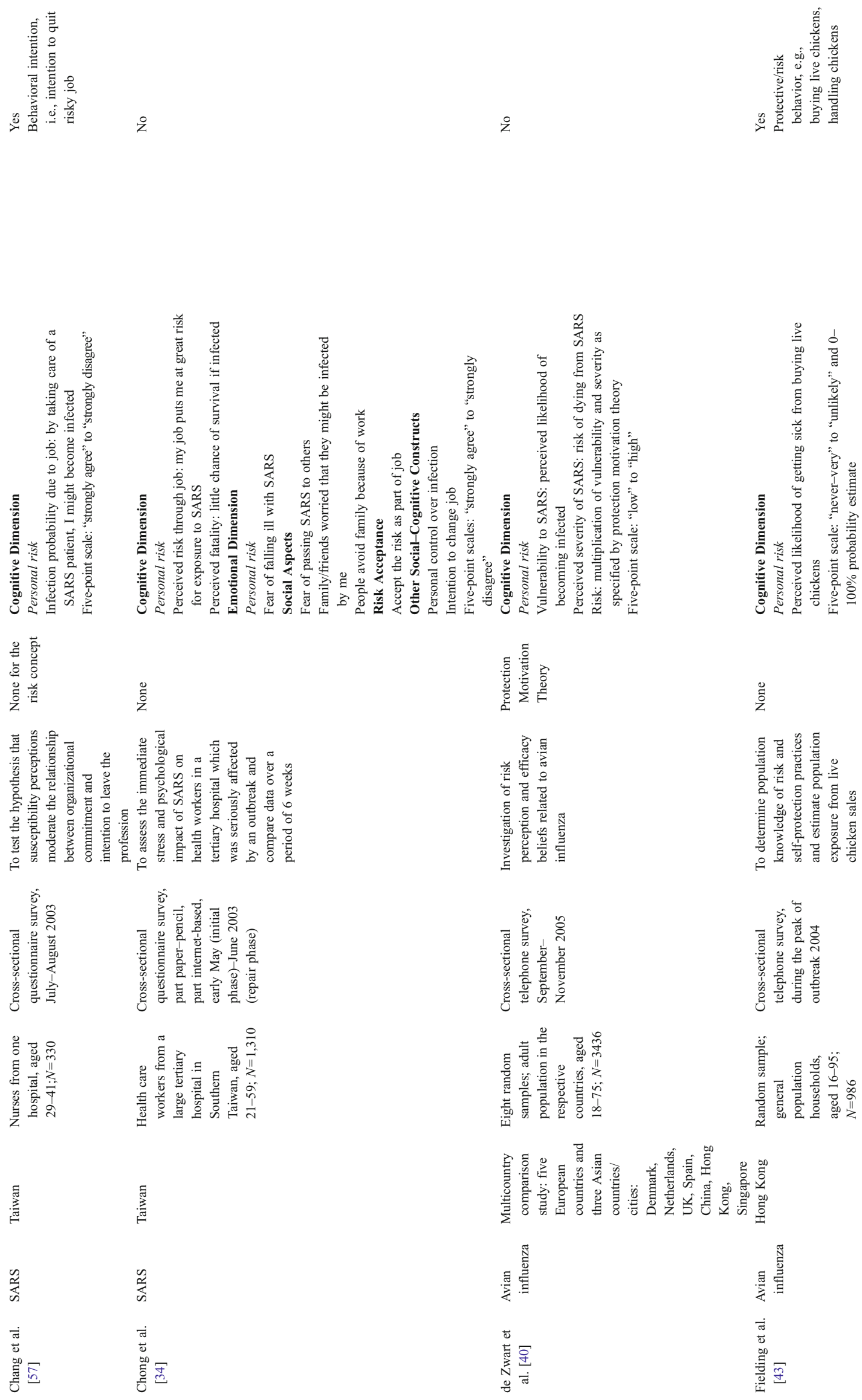


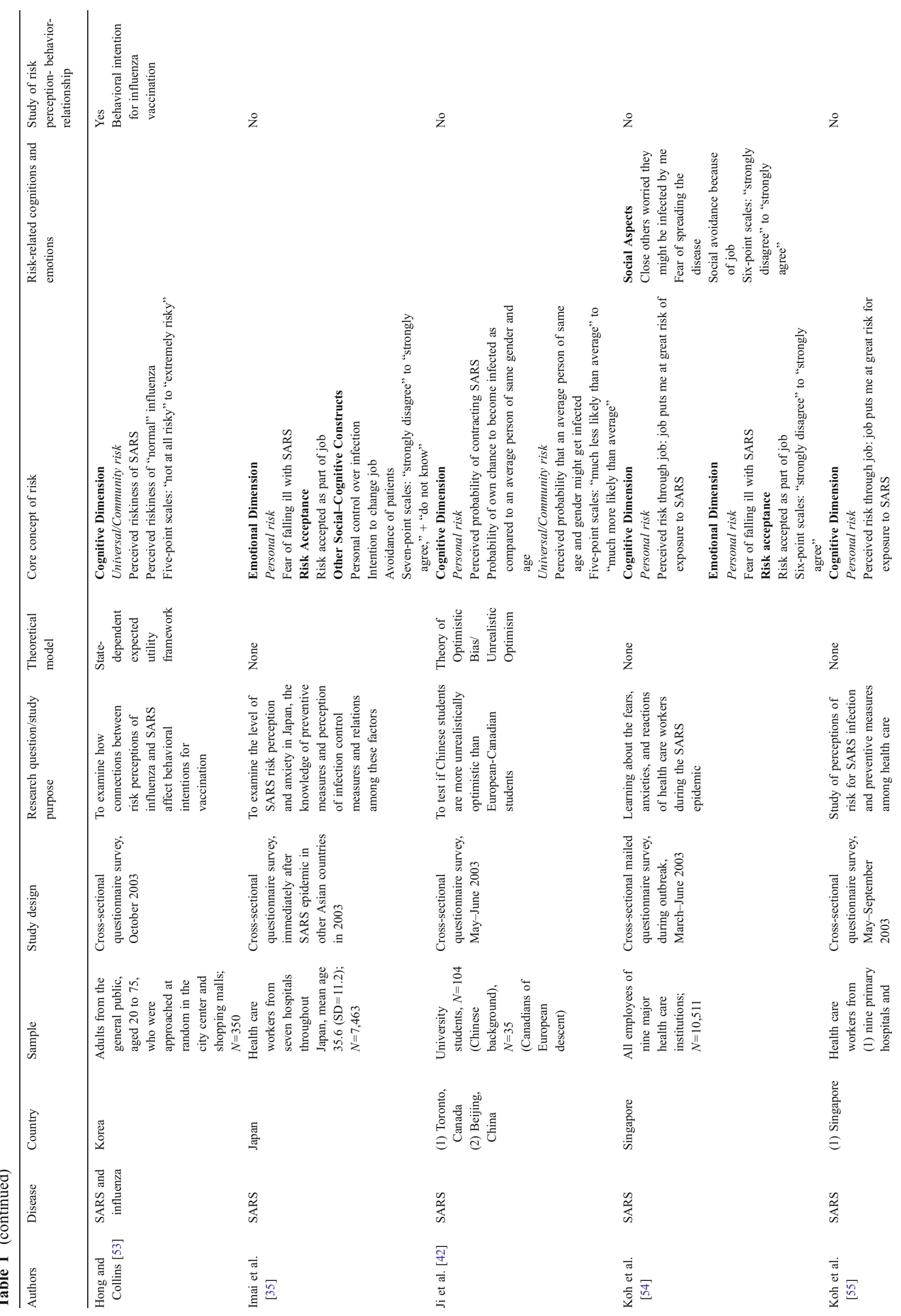




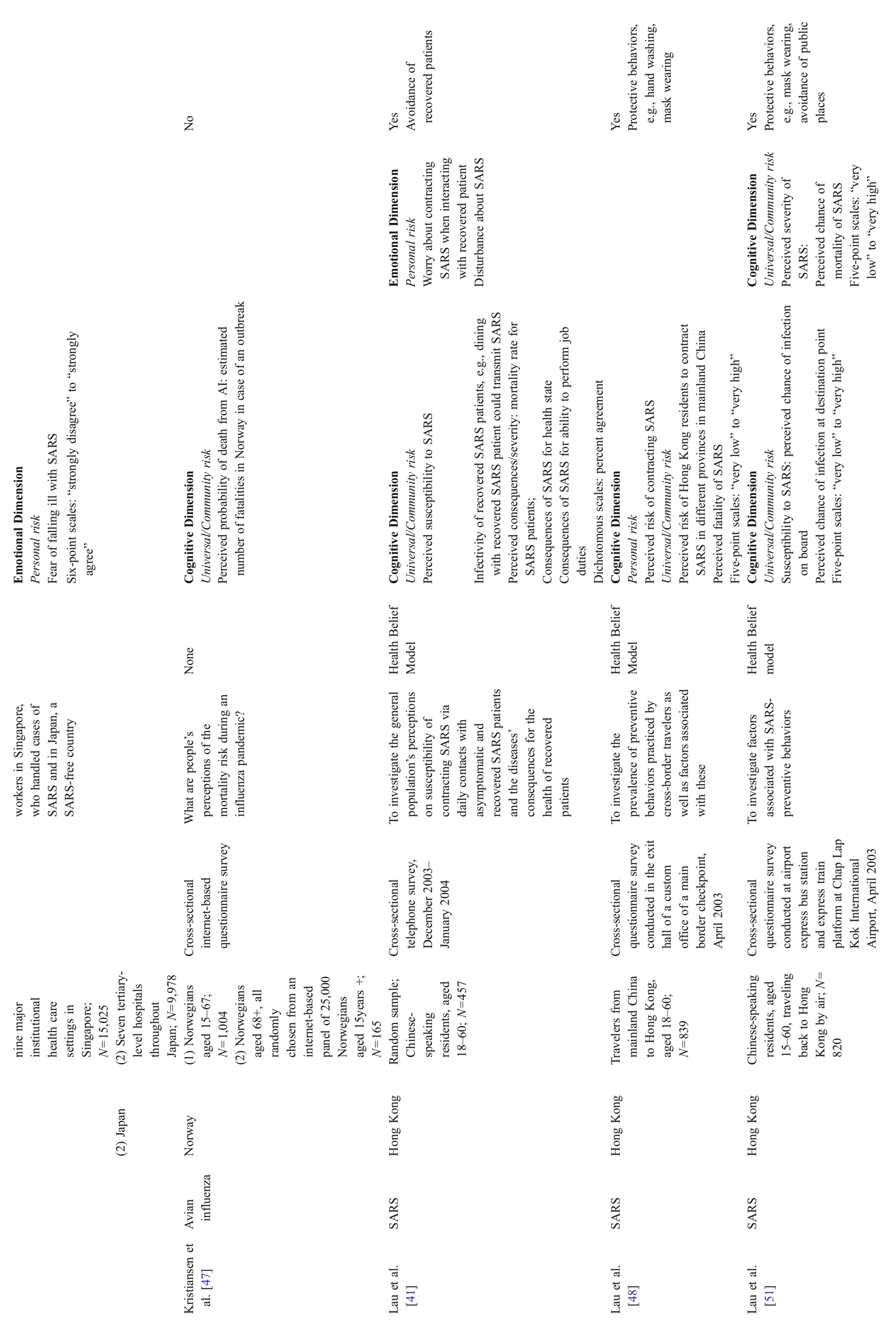




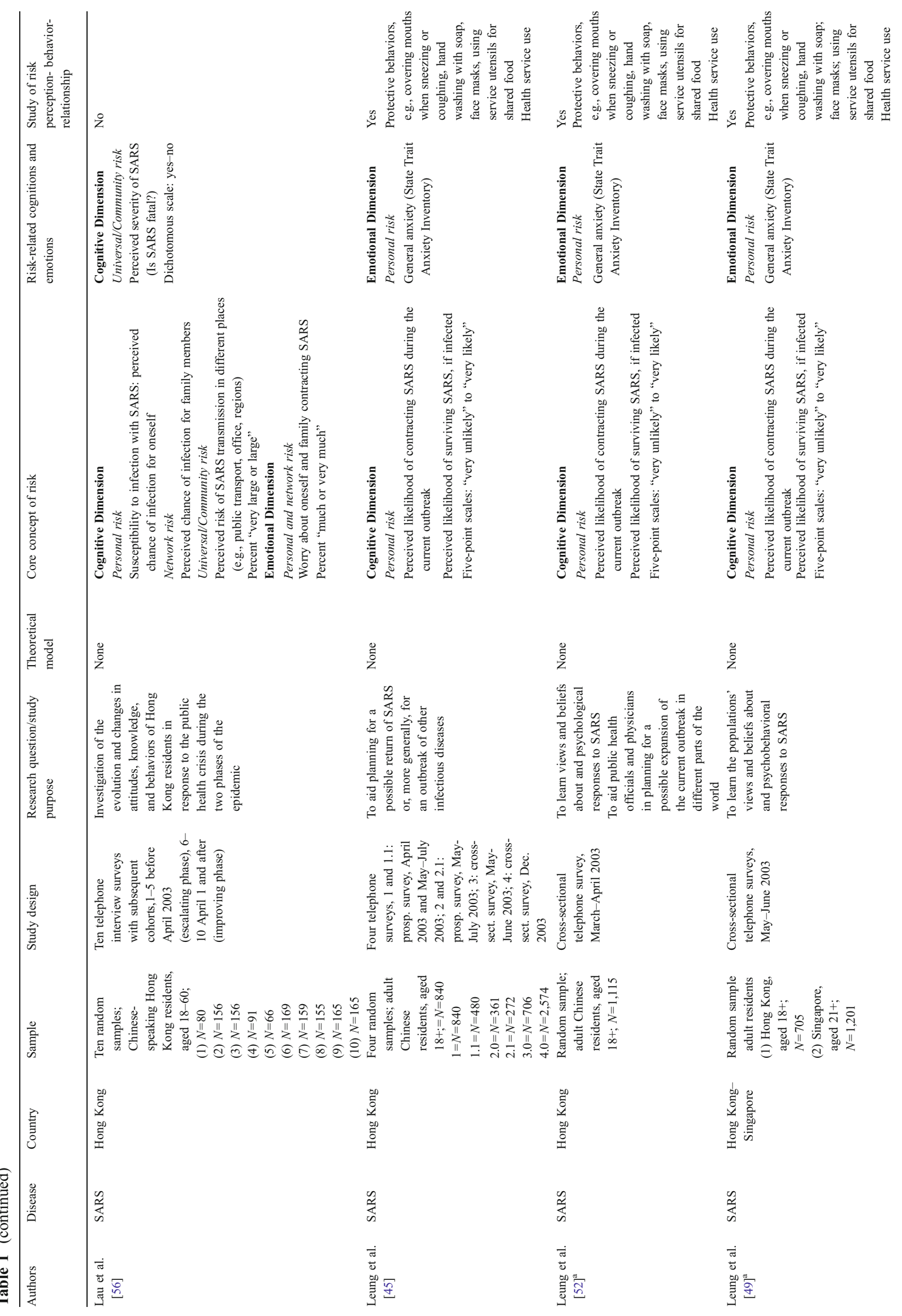


そ̊

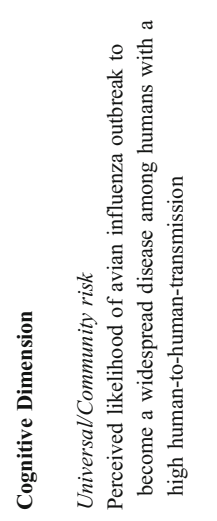

MIInWWIn

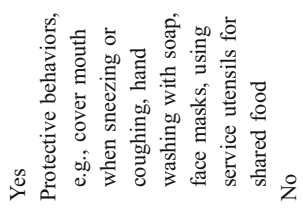

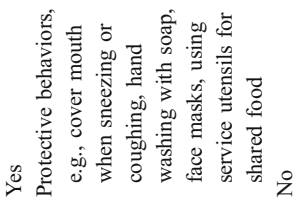
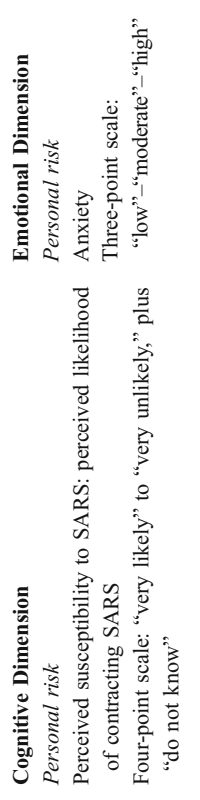

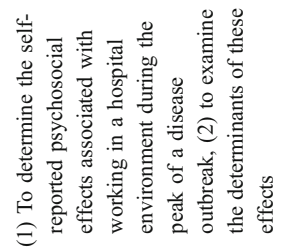

产

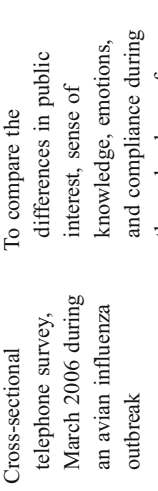

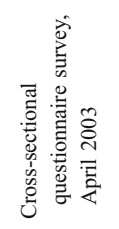

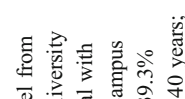

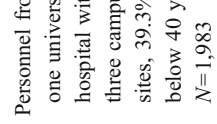

产

站

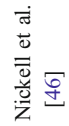

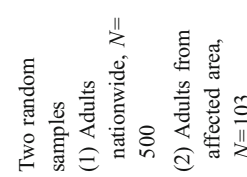

$\overline{\underline{g}}$

吝离

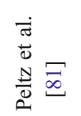
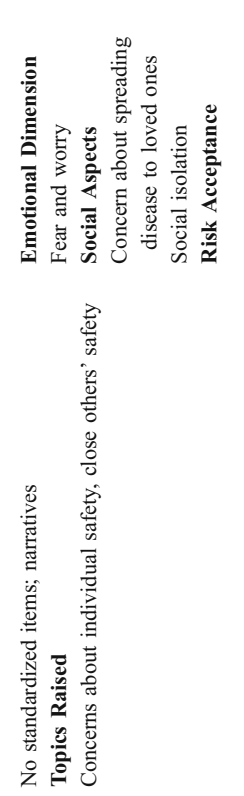

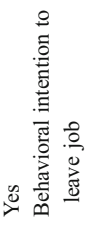

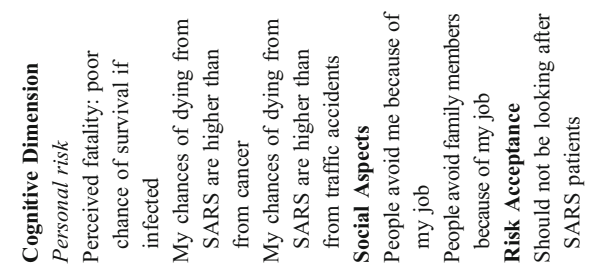

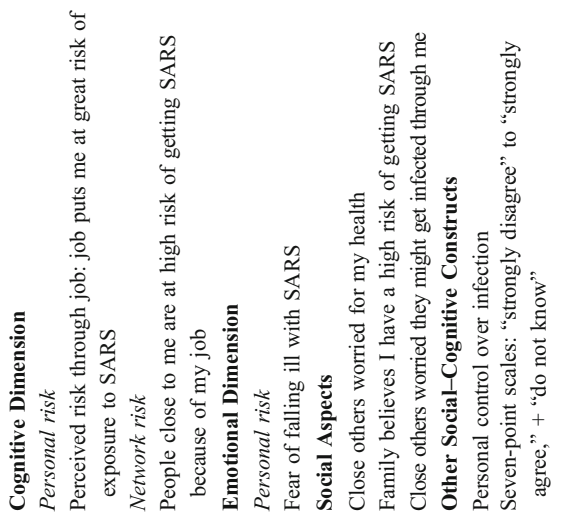

产

产

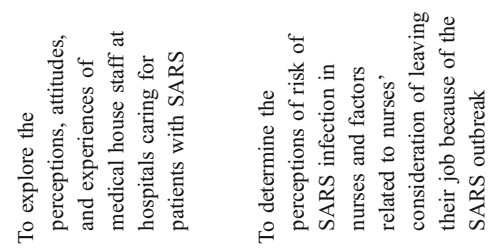

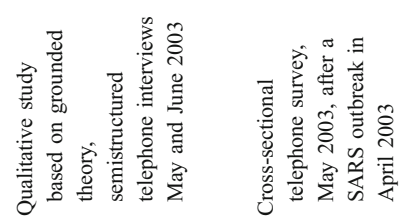

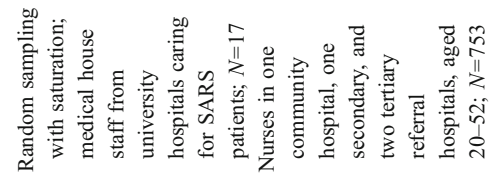

起

产

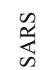

㟧

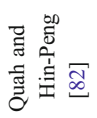

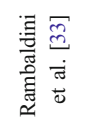

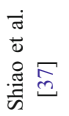




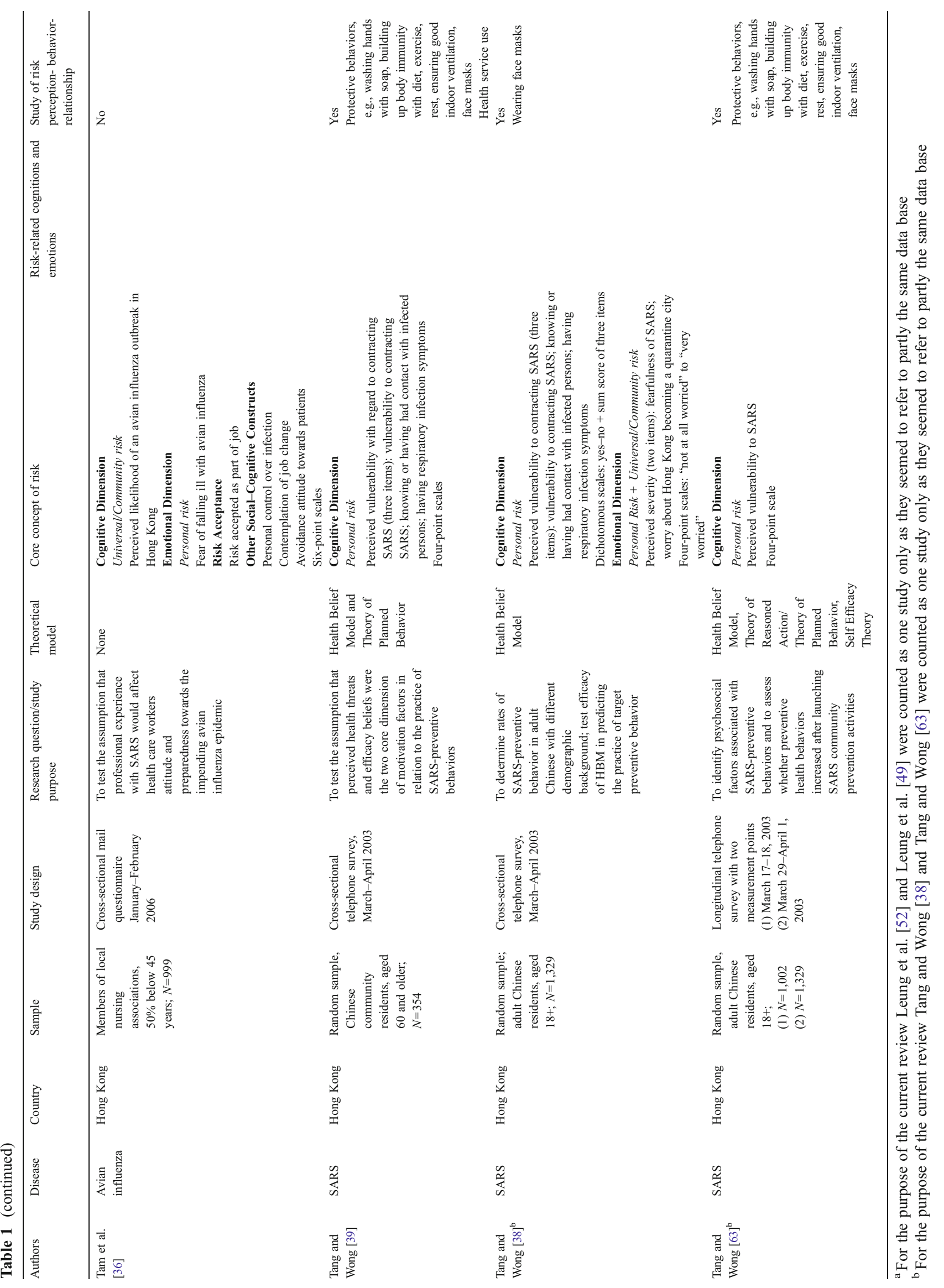


related and/or which actually were considered integral parts of risk perception by some other studies. While, for example, some authors included fatality ratings in their core concepts of risk, others presented these as independent aspects. Similarly, some studies defined emotions, such as fear or worry about SARS, within the boundaries of their risk assessment, while others also included such "emotion items" but placed them beyond the narrower realm of risk perception by labeling them as separate features.

Classification of risk concepts, as documented in Table 1 as well as in the following text, was thus carried out in two different ways. One was noting what the authors themselves explicitly referred to as their measures of risk perception, i.e., what they labeled as or listed among their risk perception measures. These will be referred to as the "core concepts" of risk perception. The other pertained to additional risk-related aspects measured in the studies. Table 1, therefore, besides listing the explicit operational definitions of risk perception in one column, contains a separate column listing those related aspects for each study.

\section{Results}

\section{Study Characteristics}

The major amount of the reviewed studies $(75 \% ; N=21)$ dealt with SARS, only $25 \%(N=7)$ focused on the relatively newer phenomenon of avian influenza. Most of the SARS studies $(90 \% ; N=19)$ were carried out in 2003 , with the large majority taking place during the outbreak in EastSoutheast Asia-Canada in the first half of that year, while the avian influenza studies were conducted at later points, between 2004 and 2006. As might be expected, most studies were carried out in those parts of the world which had been affected most, with Asia clearly in first place. Thirty-two percent $(N=9)$ of the studies originated from Hong Kong, another 36\% $(N=10)$ came from either Singapore, Taiwan, Japan, Korea, or Laos or compared samples from several of these countries. Fourteen percent $(N=4)$ came from Canada and another $18 \%(N=5)$ from Western Europe and Israel (see Table 1).

Fifty percent $(N=14)$ of the studies were based on samples from general adult populations, while the other $50 \%$ dealt with more selective groups. Mostly, these were persons at particular risk for SARS or avian influenza, such as health care workers from hospitals where SARS patients had been treated, travelers returning from mainland China to Hong Kong during the SARS outbreak, or poultry workers. Thirty-nine percent $(N=11)$ of the studies used random sampling, $61 \%(N=17)$ purposive methods to recruit their participants.
The large majority of studies $(93 \% ; N=26)$ was based on quantitative survey research designs, using mostly either standardized telephone interviews or paper-pencil approaches or internet-based surveys in a few cases. Only two studies used a qualitative approach $(7 \%)[32,33]$. Almost all studies were cross-sectional $(89 \% ; N=25)$. Only $11 \%(N=3)$ were based on a longitudinal design or used cohorts recruited at different points in time.

\section{Concepts of Risk Perception}

Not surprisingly, the analysis revealed that the measurement concepts of risk perception varied widely (see Table 1). The majority of the studies $(71 \% ; N=20)$ relied on a few single items for their core risk measures asking for perceived risk or perceived likelihood of contracting SARS/avian influen$z a$, for perceived likelihood of a SARS/avian influenza outbreak and/or perceived fatality of the disease, partly added by ratings of fear or worry with regard to the disease. Some studies, all of which investigated risk perceptions in health care workers, named a more diverse range of items under the risk perception label. Besides direct assessments of risk and/or fear of getting SARS, these included for instance perceptions of personal control, worry of close others about getting infected, and/or avoidance of patients due to infection risk or intention to change jobs [34-36, 37]. However, in these studies, too, all measures were used on a single-item basis. Two publications employed aggregate measures of perceived vulnerability (vulnerability, contact with infected persons, having respiratory symptoms) and/or perceived severity (fearfulness of SARS and worry about one's own city becoming a quarantine area [38, 39]; see Table 1).

A little more than one third of the studies $(36 \% ; N=10)$ explicitly referred to a theoretical model, mostly the HBM $(N=6)$, which also provides a definition of perceived risk (for further details, see the paragraph on the risk perceptionprotective behaviour relationship below). However, only few studies explicitly laid out their definition of risk perception in the introductory section of their papers $[32$, 40-42].

Further information on the notions of risk perception came from the exploration of the studies' assessment measures. This analysis yielded a series of categories which were then used to classify the concepts. These categories, intended to capture the major communalities and differences between the approaches rather than to represent the numerous singularities of the different studies, were: (1) psychological processes or dimensions involved in risk perception (cognitive only versus cognitive plus emotional), (2) type of judgement (expectancy only versus expectancy $\times$ value), (3) objects of risk perception (self, network, community/society), (4) type of risk (risk of SARS/avian 
influenza, risk of other health hazards, social risks), (5) risk situations (general versus situation-specific risks), (6) risk reference points on the health-disease continuum (exposure to a virus, contraction of a virus, falling ill), and (7) risk acceptance $^{3}$. Below, findings will be presented in line with these categories.

\section{Processes and Dimensions of Risk Perception: Cognitions and/or Emotions}

The distinction here refers to the different processes involved in risk experience and the resulting dimensions of the concept, i.e., whether risk was assessed in purely cognitive terms, such as asking for assessments of likelihood to contract SARS or perceived probability of dying from SARS, and/or whether risk was also measured as an emotional phenomenon, such as experience of fear or anxiety of SARS or avian influenza. A major problem with categorizing the concepts was due to the fact that a considerable number of studies $(31 \%, N=8)$ relied on the generic term "risk" for assessment (e.g., "My job puts me at great risk") mostly without adding any further differentiated rating. As the authors themselves most often seemed to treat these measures as equivalent to assessments of likelihood of contracting SARS/avian influenza, they were counted among the cognitive concepts.

Based on this assumption, $61 \%(N=16)$ of the quantitative studies chose a one-dimensional approach, the large majority of which again defined risk perception in cognitive terms only $(N=15)$. Thirty-nine percent $(N=10)$ included a cognitive as well as an emotional dimension in their core risk concepts, one of which, however, explicitly referenced the emotional assessment not to the self but asked respondents to estimate the worry about avian influenza felt by friends [43]. Terms used for the assessment of riskrelated emotions ranged from the relatively mild concern about contracting SARS/avian flu and worry about contracting SARS/avian flu to the more intense fear, which was used most often, and anxiety (see Table 1). Of the two qualitative studies, one defined risk perceptions in cognitive terms but reported also on the emotional ramifications of the risk experience [32]; the other focused on the emotional connotations of risk in terms of concern, fear, and worry [33].

\footnotetext{
${ }^{3}$ For the report on the number of studies within different categories of operationalization, it should be noted (1) that the categories are not always mutually exclusive, i.e., as many studies contained more than one measure of risk, the respective studies were also counted in more than one category, (2) that unless otherwise specified the numbers and percentages given pertain to the quantitative studies only $(N=26)$, while results of the qualitative studies $(N=2)$ will be reported separately.
}

Despite the overall dominance of the cognitive perspective on risk perception, it needs to be noted that of those studies which explicitly tapped risk perception in purely cognitive terms, six ( $23 \%$ of the 26 quantitative studies) actually also assessed emotional aspects. This, however, was done without explicitly labeling these measurements as indicators of risk perception, i.e., these were not parts of the core risk perception concepts. From this slightly wider perspective, more than half $(61 \% ; N=16)$ of the reviewed studies took account of cognitive as well as emotional aspects of risk (be it as an explicit part of risk perception or as a related concept); however, only very few explicitly dealt with the associations between these cognitive and emotional dimensions of risk. Thus, only three of the quantitative studies (11\%) gave an account of the empirical relationship between measures of cognitive risk assessment and fear/anxiety [44-46], with, for instance, one study reporting a correlation of $r=0.64$ for their measures of perceived risk and worry about contracting SARS [44]. No study provided an extended discussion about the ways in which these aspects might relate to and influence each other.

\section{Types of Judgements: Expectancy and Value Components of Risk Perception}

The single most frequently used assessment was perceived likelihood or probability of contracting the disease or perceived likelihood of an outbreak or variations of such measures which were included in $58 \%(N=15)$ of the core concepts represented in the quantitative studies. Eight studies (31\%) relied exclusively on this approach. No study took account of this type of assessment as a "side aspect," i.e., explicitly placed it beyond the risk label. In contrast, another type of probability rating, i.e., fatality ratings or ratings on probability of death/survival was apparently considered less central. While $46 \%(N=12)$ of the studies included a measurement of perceived fatality, only $35 \%(N=9)$ mentioned this aspect in their core construct of risk, two of which defined risk exclusively as perceived fatality [46, 47]. None of the reviewed studies conditioned their probability assessments on anticipated or actual behavior change.

Compared to the expectancy component, the value or severity element of risk seemed distinctly underrepresented, as the overwhelming majority of studies did not request explicit ratings of severity from their participants (e.g., "How severe or serious is SARS?"). If, however, all the studies measuring fatality as part of their core risk construct were also counted as assessing severity $(35 \% ; N=9)$, the picture seemed a little less skewed. Yet, even then, only seven, i.e., $27 \%$, of the quantitative studies assessed both likelihood of contracting as well as severity/fatality as parts 
of their core risk concept [38, 40, 41, 44, 45, 48, 49]. When those studies which assessed both likelihood as well as severity/fatality outside of their explicit risk measures were included $[37,50,51,56]$, the percentage rose to $42 \%(N=11)$. However, only one study used the multiplicative term between the two ratings, as specified by expected utility theory and its health-related applications such as the HBM or PMT [40].

\section{Objects of Risk Perception: The Self, Network, and Community}

Another obvious difference in conceptualizing risk perception was related to the risk object chosen, i.e., who the risk was assessed for. Universal risk, which could also be perceived of as societal or community risk, refers to a global perception of outbreak probability or general fatality rates in case of an outbreak in a specific area. Personal risk perception and network risk perception, on the other hand, are estimations of one's own and close others' very specific probability to contract SARS or avian influenza or to die from this disease as, for instance, specified in the HBM or PMT. Forty-six percent $(N=12)$ of the 26 quantitative studies focused on the personal perspective alone, i.e., assessed the risk for the self without tapping universal risk in their core concepts, while $27 \%(N=7)$ measured solely universal or community sense of risk, and another $27 \%(\mathrm{~N}=7)$ looked at both aspects. Assessment of network risk was less common and occurred exclusively in combination with either of the other two measures. Five of the quantitative studies (19\%) asked respondents to rate risk for close others, either within or beyond their core risk concepts, and both qualitative studies also reported concerns of respondents about the risk/safety of their loved ones.

\section{Type of Risk: Different Health Risks and Social Risks Accompanying Health Risks}

Three, i.e., $11 \%$, of the quantitative studies included measures which, besides asking for risk related to SARS or avian influenza, also tapped risk related to other diseases or requested direct comparisons between perceived risks for different diseases [37, 44, 53].

Another three studies (11\%), all of which were looking at perceived risk in health care workers, also included some measure on social aspects of risk, such as perceiving a risk of spreading the disease to others $[34,54]$ and/or on whether people believed that their close others felt worried about their health due to the respondent's job in the health care sector [34, 37, 54]. This issue of perceived risk to transmit the disease and ensuing role conflicts between professional and family obligations was also raised by the qualitative studies ("All residents expressed concern about the potential risk...they posed to their loved ones" [33], p. 382) [32].

The same studies raised another issue which might be relevant in this regard, even if it was not explicitly claimed as part of an anticipated "risk" because it mostly referred to actual past experiences, was that of possible social isolation and stigmatization due to having a job in the health care sector [34, 37, 54]. Again, one of the qualitative studies also reported that respondents raised the issue of felt isolation due to their exposure to the virus ("The cancellation of rounds and the restrictions placed on social interactions with other health care workers created a sense of isolation" [33], p. 383).

\section{Situational Specificity of Risk Appraisal: General Risks and Specific Risks}

Variance occurred also in terms of the generality or specificity of risk assessments. Forty-two percent $(N=11)$ of the quantitative studies phrased their questions in unconditional/absolute terms (e.g., "likelihood of contracting SARS"), whereas $58 \%(N=15)$ asked for qualified/conditional statements (e.g., "likelihood of contracting SARS in place x" or "likelihood of contracting SARS when doing y") or included both aspects. In particular, these ratings pertained, for instance, to the probability of contracting SARS in different places within a city, such as public places, offices, or public transport [56], when traveling on board of a plane [51], when interacting with recovered patients [41], or risk from specific behaviors such as buying live chickens [43]. Specified assessments were also requested in most of the studies which investigated risk perceptions in "professional risk groups," such as poultry workers or - primarily employees in the health care sector $(23 \% ; N=6)[34,37,50$, $54,55,57]$. Both qualitative studies as well reported on risk perceptions related to a job in the health care professions $[32,33]$. Yet another differentiation occurred within the group of studies which were conducted outside of acute outbreak areas and/or during times without an outbreak. While most studies - just like those conducted in outbreak areas - asked for general risk assessments, an alternative was to explicitly link the measurement to outbreak occurrence (i.e., likelihood of contracting or dying from the disease, if one's own country were hit by an outbreak [47]).

\section{Risk Reference Points on the Personal Health-Disease Continuum: Risk of Exposure, Risk of Infection, and Risk of Illness}

Differences in the core risk concepts also became evident in terms of how perceived risk was specified in relation to the reference points along the personal health-disease contin- 
uum. The most common choice among the studies which in any form assessed personal risk was to ask for likelihood (or else risk or fear) of infection or for contracting or getting SARS/avian flu $(N=10 ; 38 \%$ of all quantitative studies). A few studies $(N=4 ; 15 \%)$, however, in slightly different terms asked for ratings of risk of exposure to SARS/avian flu [34, 37, 54, 55], and instead or in addition requested respondents to indicate their fear or likelihood of falling ill or fear of getting sick $(N=7 ; 27 \%)[34-36,37$, $43,54,55]$.

\section{Risk Acceptance}

Finally, five $(19 \%)$ of the quantitative studies introduced yet another feature (four of them explicitly as part of the risk construct), asking health care workers whether they accepted risk as part and parcel of the job [34-37, 54]. Rambaldini et al. [33] in their qualitative study on health professionals' reactions to the SARS outbreak also reported on this aspect of risk perception.

\section{The Risk Perception-Protective Behavior Relationship}

More than half, i.e., $57 \%(N=16)$ of the 28 reviewed studies investigated the role of risk perception for behaviors or behavioral intentions aimed at avoiding infection. Mostly, these were individual hygiene or lifestyle behaviors or behaviors related to handling live chickens $(N=11$; $39 \%)$. A few studies $(N=3 ; 11 \%)$ dealt with social avoidance behaviors/intentions, such as health care workers intentions to leave their job $[37,57]$ or tendencies in the general public to avoid recovered SARS patients [41]. One study investigated behavioral intentions for influenza vaccination as a result of SARS risk perception [53] and another compliance with quarantine [32].

The remainder of the studies $(43 \% ; N=12)$ was either of a purely descriptive-epidemiological nature or had their focus on potential determinants of risk perception. Of these, only two (17\%) explicitly based their risk concepts on a theoretical model, i.e., the PMT [40] and Weinstein's [58] Optimistic Bias concept [42]. This low percentage of model-based research increased to $50 \%(N=8)$ in the subgroup of studies which investigated the potential role of risk perceptions as determinants of health-related behaviors or behavioral intentions $(N=16)$. Of the seven quantitative studies which were model-based, three used the HBM [41, 48, 51]; two further studies [38, 39] named the HBM as well as the Theory of Reasoned Action/ Theory of Planned Behavior [28, 29] and Bandura's Self Efficacy Theory [59] as the basis of their work. Another choice presented by one of the studies [44] was the Precaution Adoption Model [60], and one further study [53] based its research on a state-dependent expected utility framework $[61,62]$. In addition, the HBM and PMT were claimed by one of the qualitative studies, which explored the association between risk perception and compliance with quarantine [32]. In three of the quantitative studies all or most of the variables introduced by the respective model were tested within a multivariate analysis $[39,48,51]$, while the other studies used major components of the theories and/or tested bivariate relationships between separate model components and protective behaviors. Only one of the model-based studies had a longitudinal design [63].

\section{Discussion}

In the following section, the theoretical concepts of risk which emerged from the reviewed articles as well as the role risk perceptions play for protective behaviors will be summarized and examined. Strengths and deficits of current empirical risk perception research in terms of their theoretical foundations will be pointed out and conclusions will be drawn regarding implications for future research and also for practical purposes such as the development and design of public health risk messages.

\section{Concepts of Risk}

The majority of the reviewed studies were launched as a rapid response to the SARS outbreak in 2003 or as an immediate reaction to the first human cases of avian influenza in some Asian countries. The purpose was thus socioepidemiological in most cases, aimed at assessing psychological reactions towards the SARS or avian influenza threat in general populations or in specific subgroups, such as health care workers, to predict adoption of protective behaviors and thereby to provide data for future intervention planning. This predominantly practiceoriented intent might explain the pervasive lack of conceptual elaboration on the perceived risk concept that was encountered. In fact, only few studies explicitly defined or theoretically explained their notion of perceived risk. Instead, it was mainly the operational level, i.e., the measurement instruments, which provided indications about the underlying notions of risk perception.

The concepts emerging this way turned out to be quite heterogeneous - and this finding related to a whole number of features characterizing the risk concept, including: the psychological processes or dimensions involved in risk perception (cognitive only versus cognitive plus emotional), type of cognitive judgement (expectancy only versus expectancy $\times$ value), objects of risk perception (self, network, community/society), type of risk (SARS/avian influenza risk, other health risks, social risks), risk 
situations (general versus situation-specific risks), risk reference points on the health-disease continuum (exposure to a virus, contraction of a virus, falling ill), and risk acceptance. The variance which occurred on all these dimensions clearly indicated that behind the common claim to investigate "risk perception" there was only very limited agreement on what exactly constitutes such perception of risk and how it should be measured. This, however, suggests that the empirical findings from the different studies on risk perception might be extremely difficult to compare and evaluate within a common context.

One of these differences, which actually touched the very core of the construct, became evident with regard to the choice of defining risk perception as an exclusively cognitive or as a cognitive-emotional phenomenon. While it seems plausible that a pandemic influenza outbreak constitutes a highly uncertain, ambiguous, and threatening situation, where lay people tend to fall back on immediate, visceral, and emotionally loaded concepts and images rather than rational probability assessments, this notion needs empirical testing: at different points in time, before and during an outbreak, and with different subgroups within general populations. However, only a little more than half of the reviewed studies looked not only at cognitive risk assessment but also at SARS- or avian-influenza-related emotions. Only about one third of the quantitative studies explicitly perceived of this emotion-based judgement as a dimension of risk perception and not as some external aspect. And whatever the concept - "risk as feelings" or "risk and feelings" [19, 64] - neither of the studies provided further theoretical elaboration on why fear or worry were perceived of as part of risk perception or seen as separate features, just as there was little interest in the empirical relations between cognitive and emotional aspects of risk perception. At this point, empirical research on SARS and avian influenza risk perception can thus provide few insights into the issue of whether emotions result from cognitive risk assessment and/or precede such cognitions or how cognitive and emotional processes interact in creating notions about "risk" and influence behavior. While the mostly social-epidemiologically oriented studies which formed the basis for the current review were not designed and intended to investigate such issues, future research should be directed towards this area as findings would not only be relevant for theory development but also for the ways in which health risk messages will have to be framed and designed in case of future outbreaks.

Another notable issue was the considerable heterogeneity in the operational terms chosen to measure risk affect, which ranged from the relatively mild concern and worry to fear or anxiety. Affirmative responses will probably be stronger for worry or concern than for anxiety, which makes these findings difficult to compare and evaluate. Also, while worry and concern can be perceived of as cognitive aspects of emotion [65, 66], anxiety and particularly fear, might tap more strongly into an affective-visceral dimension. These aspects are likely to relate differently and interact differently with cognitive probability ratings of disease [66], but that notion, too, requires empirical testing.

Another difference concerning the essence of the risk paradigm became apparent in the way in which the studies measured the type of risk judgements, i.e., whether they assessed risk in terms of the expectancy and/or the value component. What came as a particular surprise was that only few studies actually relied on the theoretically established "probability $\times$ severity function" which posits that people's choices are a product of assessments of probability and utility/desirability of options [2, 5-8]. First, quite a number of studies did not use a differentiated concept of risk at all but simply asked respondents to rate their risk to contract SARS. While this type of assessment, particularly when related to terms like "contracting" or "getting" a disease, can be considered predominantly cognitive and conceptually close to the "likelihood of contracting-measure," there is also plausibility in the assumption that the term risk inherently entails severity aspects and/or is likely to incur more negative emotional connotations than the more neutral likelihood and is therefore conceptually closer to "fear." This is also supported by psychometric paradigm-related research, suggesting that lay people tend to judge events with low probability but high catastrophic potential such as nuclear accidents as "high (dread) risk" [3, 4].

Furthermore, while probability of infection or of contracting disease was clearly the most common notion of risk perception, the value - or severity - component met with considerably less interest. However, a substantial number of studies included measures of fatality and it might be argued that fatality ratings are in fact assessments of value (i.e., severity) rather than measures of expectancy. Indeed, in many studies which used fatality measures, these were explicitly introduced as measures of severity of disease. Such a claim certainly has some degree of content validity, since most people would consider diseases they believe to be potentially fatal also as serious, even if from a purely structural-systematic perspective, probability of death ratings clearly refer to an expectancy-, not to a value-element. However, even when these ratings were counted as measuring severity, it seemed that severity was not generally considered to be an integral part of the risk concept, but was more often presented as a separate entity. This was also emphasized by the fact that only one study actually used the multiplicative term specified by the HBM and PMT [40], i.e., the product of probability and severity. All these deviations from standard definitions of risk occurred mostly without any explanation of the rationale behind them - 
even in cases where models such as the HBM or PMT had been referred to in the theory sections of the papers. Testing the effects of subcomponents and their possible interactions, however, might yield essential information for effective risk communication, for instance, in terms of being able to specifically target unrealistically low perceptions of occurrence likelihood versus underestimations of severity.

Further variance was encountered in terms of the objects risk was rated for - a problem that Brewer et al. [26, 27] have pointed out and which also became apparent in the present review. The most common assessment related to the self (personal risk), but some studies also measured risk perceptions for close others (network risk) and/or for the society/community as a whole (universal or societall community risk). While network risk was commonly measured in addition to personal risk, societal/community risk was most often assessed instead of personal risk. While for respiratory infections risk of self and risk of others - due to "social contagiousness" - are bound to be correlated, they are also liable to differ. Judgements on personal likelihood to contract infectious disease are subject to considerations of individual immune competence and host resistance. Since diseases such as SARS and avian influenza are still relatively new and unknown in the wider population, people are likely to refer to personal experiences with diseases which might seem to bear some resemblance to "normal" flu or other infectious diseases. Based on differing individual experiences with susceptibility or immune competence, there could be considerable divergence between personal ratings and those for the population in general. In addition, tendencies towards an optimistic bias [58] should influence personal ratings to be generally lower than those for the overall population. Harris and Middleton [67], who investigated risk perceptions for various health hazards in students, showed a gradient for most of the rated conditions depending on who the rating was made for: from lowest ratings for the self to slightly higher ones for very close others, even higher ones for acquaintances, to highest scores for unknown others. Even if such tendencies should be less pronounced for respiratory infections such as SARS, which - compared to lifestyle disorders - tend to be "equalopportunity diseases," subjective concepts about individual susceptibility or resistance capacity might produce quite different results depending on who the risk is assessed for. In any case, if the goal is to determine how risk perception influences protective behaviors, personal risk assessment should be the first choice, while the other perspectives while also important - should provide additional rather than surrogate insights.

Surprisingly few studies extended their view from the health dimension of SARS or avian influenza risk to other types of risks. Thus, only three studies asked their respondents to also rate other disease risks or to make direct comparisons between risk of SARS and other risks [37, 44, 53]. Such comparisons could provide important insights, as the motivational potential of risk perceptions in case of an outbreak depends not only on their absolute but also their relative strength when compared to other health concerns. Relative importance of pandemic influenza risk, however, also relates to other types of risk which might be involved in a pandemic influenza hazard such as, for instance, social risks. Especially studies looking at health care workers did assess not only personal health risks but also the perceived risk of spreading the disease to family and friends and/or issues of stigmatization and social isolation [32-34, 37, 54, 55]. While in the reviewed studies stigmatization and social isolation were mostly assessed in terms of actual experience, it is plausible to assume that anticipations of such processes ("social risk cognitions") are relevant for shaping the sum of pandemic influenza risk connotations and thereby influences behavioral responses. Above and beyond risk to one's personal health, anticipations of being responsible for spreading the virus to loved ones or being socially excluded because of one's job might detract considerably from readiness to accept risk and might interfere with professional behavior in health care workers. This issue was also explicitly reflected in the concept of risk acceptance, which was brought up by a few studies [33-36, 37, 54, 55]. How professional groups who have a key role within containment plans balance personal and social risk expectations with their professional roles, how they weigh the risk of getting infected and in turn infecting others against the risk of jeopardizing their professional identity, is a concern which needs to be faced and dealt with in the course of effective intervention planning. Otherwise, a sudden lack of readiness of professionals will disrupt care for those who have already become patients or get in the way of public health containment measures and endanger collective protective action.

Social considerations are, of course, not restricted to professionals, as Yiang et al. in a contribution to the current issue, have shown. They investigated determinants of risk perception within Chinese communities in the Netherlands and the UK, pointing out the role of social costs of infection due to anticipated stigmatization processes. Similarly, a recent qualitative study on Hong Kong SARS survivors [68] showed that stigmatization and discrimination was a pervasive and traumatic experience, a finding which led the author to conclude that anticipations of such social exclusion processes might well endanger the effectiveness of public health measures should there be another major outbreak (see also [69]). Particularly in the early stages of an outbreak, when people are perhaps anyway more ready to interpret ambiguous respiratory symptoms as indications of "normal" flu than of a life-threatening disease, they might thus deliberate and delay, hoping that the symptoms 
will turn out harmless and go away, rather than take the risk of being prematurely identified as a carrier of a potentially lethal virus. Social risk perceptions such as these could easily cancel out motivating effects of perceived disease risk. Thus, the so far most commonly used one-dimensional notion of SARS/avian influenza risk as a "mere" health hazard might fall short of grasping the whole of what makes up lay persons' or health professionals' ideas about pandemic influenza risk.

Another angle which remains to be investigated by future studies is the relative relevance of health risk perceptions compared to perceptions of other types of life risks. In one of the original contributions to the current issue, Voeten et al. reported lower avian influenza risk perceptions in a sample of Chinese residents in the UK and the Netherlands in comparison to the general populations in these countries. According to the authors, an explanation for this rather surprising finding might be found in studies such as the one by Lai and Tao [70] who showed that Chinese people in general seem to be far less concerned about disease risks than about risks that threaten, for instance, national stability and economic development. In the same vein, two of the reviewed studies [40, 42] reported lower risk perceptions in Asian as compared to European or North American samples. While this variation may largely be due to social-cultural differences, socioeconomical considerations may also play a part. Thus, people living under economically precarious conditions may place a relatively lower value on health consequences, at least as long as these remain speculative and distant when compared to other more immediate economic daily hazards. This might be specifically relevant for groups such as poultry workers who in case of an avian influenza outbreak will find themselves between a rock and a hard place when evaluating health vs. economic risk. Vice versa, as an example from the other end of the socioeconomic spectrum, globally mobile executives might at some point have to face the "bads" of globalized risk society as the almost inevitable flip side of the "goods" [71, 72], having to weigh the risk of health damage against that of economic losses due to restricting travel. Future research will have to take up the challenge to understand the processes by which pandemic influenza risk is grounded in the (sub) cultures and social structures of different countries and their respective worldviews [4] in order to effectively tailor communication strategies to specific groups, particularly those who might face a heightened risk of exposure to influenza viruses while at the same time facing economic or social risks, like for example poultry farmers and vendors in the case of $\mathrm{H} 5 \mathrm{~N} 1$.

As for the generality or specificity of risk situations, a little less than half of the studies asked for general risk assessments, i.e., overall probability to contract SARS or general fatality rates, while a bit more than half qualified their assessments in one way or another. What was requested were, for instance, risk ratings for specific regions or ratings for situational circumstances, such as probability of infection when traveling or due to working in a specific job such as in the health care sector. Particularly, behavior-specific risk ratings should be expected to relate more closely to particular protective behaviors than general risk perception. In an outbreak situation specific assessments can help identify "critical areas of risk perception" where people perceive unrealistically low or high risk and which might warrant intervention. Vice versa, general risk expectations provide an interpretational background for behavior-specific risk perceptions because, even when specific risks are perceived of as not particularly high, this does not necessarily imply that risk perception in general is also low and vice versa. Therefore, both types of risk perception are essential and should not be treated as surrogates.

Yet another type of qualifying assessments became apparent in the small group of studies which were conducted outside of acute outbreak areas. It should make a difference whether respondents are asked for general statements ("probability of contracting SARS/avian influenza") or whether such ratings are conditioned on the case of an outbreak ("probability of contracting SARS/avian influenza if an outbreak occurred in the country/region"). The latter should yield higher risk perceptions than those without qualification, as in the former case the disease has yet to arrive and might seem not only geographically but also psychologically more removed. In general, hypothetical risk perceptions such as these are hard to compare to "real" risk perceptions under outbreak conditions and do not refer to exactly the same phenomenon. Unlike chronic diseases, which develop gradually, pandemic influenza tends to be a more binary or "onoff phenomenon", which sets quite distinct frames for risk perception under conditions of outbreak versus no outbreak. It would be profitable to study how hypothetical risk perceptions in a non-outbreak situation develop under conditions of imminent threat and whether perceptions of hypothetical risk can be extrapolated to actual risk perception. This, of course, would require introduction of long-term continuous surveillance studies.

Finally, variance also occurred with regard to the reference point chosen for assessment on the health-illness continuum. While most studies measured "likelihood of contracting SARS/avian influenza," there were also some which asked for ratings of "exposure to SARS" or "falling ill with SARS/avian influenza." Such diverse semantics could evoke different connotations. Exposure occurs prior to actual contraction or acquisition. Health care workers in an outbreak area might perceive a considerably higher likelihood of exposure to pandemic influenza than of actually contracting the disease because they might hope that immune competence and control measures would 
protect them from actually being infected. And while as a rule contracting the virus will also mean falling ill with the disease, the latter expression seems to indicate a step forward on the semantic continuum and to evoke stronger emotional connotations than a more neutral rating on probability to contract pandemic influenza.

\section{The Risk Perception-Protective Behavior Relationship}

As outlined in the introductory section of the paper, much of the research work on the health risk perceptionprotective behavior relationship has been characterized by conceptual as well as methodological problems, leading to substantial interpretational ambiguity. The specific set of papers on SARS and avian influenza risk perception reviewed here seems to make no exception in this regard. Just as there was little theoretical elaboration on the risk concept itself, only a minority of the subgroup of studies which investigated the possible predictive role of risk perception for protective behaviors could actually be considered model-based. The current review showed that, of the 16 studies which were dealing with this issue, only half referred to any theoretical concept or model, most often the HBM. Of these, again only less than half actually tested the full respective model.

The resulting lack of opportunity for studying the complexity of the decision-making process is bound to also affect the extent to which findings for the relationship between risk perception and protective behaviors can be interpreted. To obtain a comprehensive picture of how decisions about protective behaviors are made, risk perceptions need to be tested in comparison to and/or in concert with other cognitions, such as those specified by the HBM or PMT, i. e., perceived efficacy of countermeasures (outcome expectancies) or the costs and benefits associated with behavioral change. For instance, the social aspects of pandemic influenza risk discussed above could also be conceptualized in terms of costs associated with risk-related behavior, as specified by the HBM. Thus, as outlined above, the cost factor "anticipated stigmatization" (or "social risk perceptions") might neutralize mobilizing effects of health-related risk perceptions, for instance by precluding disclosure of symptoms. Also, to answer the question "how high is high enough" with regard to risk perception and "how high is too high" because helplessness sets in, risk cognitions would have to be jointly investigated with their emotional connotations and also together with outcome expectancies about control measures as well as self-efficacy expectancies. Taking action is bound to depend not only on motivating factors such as risk perception but also on enabling features like perceived availability of effective behavioral options or perceived social norms. Also, the construct of risk acceptance introduced by a few studies might further contribute to successfully predicting protective behaviors. Even high health risk perceptions in terms of likelihood to contract an infection might fail to have mobilizing effects if the related social risks are given greater weight, so that the health risk, even if high, is tolerated. Thus, a more systematic application of multifactor models would allow for far more complex insights into the workings of risk perceptions in shaping behavior than the most often used tests of bivariate relationships alone.

In addition, the utility of the data presented by the reviewed studies might be constrained by design deficits of the studies and ensuing validity problems. Despite their claim to investigate the motivating effects of risk perception on health-protective behaviors (behavior motivation mod$e l$ ), the overwhelming majority of the studies which looked at risk perception-protective behavior relationships actually investigated risk perception-behavior accuracy models [26]. That is, due to the dominating correlational study designs, positive relations between risk perception and behaviors, like hand washing, wearing of face masks, or being careful in handling live chickens, indicate little more than the fit between perceptions of own protective behavior and risk (accuracy hypothesis), as people who protect themselves are also more likely to perceive less personal risk because of these protective behaviors. This ambiguity was exacerbated by the fact that no study attempted to compare behavior-conditioned and behavior-unconditioned risk perceptions, i.e., looked at risk perceptions with or without anticipated or past behavior change, as suggested by Brewer et al. [26, 27]. Not taking this precaution can lead to dilution of risk perception effects, as some respondents might already factor in behaviour change when estimating their personal risk.

On the other hand, one could argue that pandemic influenza constitutes a special case. The investigation of this type of hazard might make studies less susceptible to internal validity threats than either studies on chronic disease or on types of acute disease which can be offset by vaccination, as in the Lyme disease example used by Brewer et al. [26]. Unlike the individual long-term development of chronic disease (e.g., coronary heart disease), where there is an extended time-frame for behavior-change, an outbreak situation creates a massive, collective, and acute threat, i.e., it has "dread potential" in terms of the psychometric paradigm, and people tend to feel more out of control $[3,4]$. It seems likely that in the first stage of an outbreak risk perception is strongly influenced by the nature of the hazard and the way it is communicated by media coverage of case and fatality incidents. That impact might be more overwhelming than any effect which countermeasures might have on risk perception. In fact, the few longitudinal or cohort studies in this review found that during the initial phases of the SARS outbreak in 2003 there was a steady increase in risk perception, which only 
leveled off in later phases [45, 56]. Moreover, unlike other types of infectious diseases, which can be neutralized by almost $100 \%$ effective vaccinations, in case of pandemic influenza people cannot be certain about the effectiveness of preliminary vaccines or behavioral containment measures, so that it is unlikely that even strict adherence to protective behaviors will nullify any notion of risk. However, while these characteristics of pandemic influenza risk might lessen interpretational ambiguities, it will not eliminate them, particularly when studies are conducted in the latter phases of an outbreak. As consistent behavior change is more likely to have occurred by then, it is also more likely that behavior motivation effects are wiped out or reduced by appraisal effects (lower risk perceptions due to higher protective behaviors). Studying the change of pandemic influenza risk perceptions and their "true" influence on protective behaviors over time therefore remains an important objective, requiring not only studies with longitudinal designs, starting after potential future outbreaks, but also long-term surveillance studies which are initiated before an actual outbreak occurs.

\section{Limitations}

It certainly needs mentioning that the classification of studies into the different categories can be disputed in some regards, due to the problems outlined in the "Method" section. Thus, for instance, to categorize the studies which directly asked for ratings of "risk" (e.g., "My job puts me at great risk") into the category of cognitive risk judgements, analogous to the measurements of likelihood or probability of infection, can be considered problematic, as the term "risk" is affectively less neutral than "likelihood" or "probability". Also, in general, the descriptions of measurement instruments in the reviewed articles were not always sufficiently precise to permit completely clear-cut categorization. Similarly, it cannot be ruled out that to some extent the differentiation into "core concepts of risk perception" and "side aspects" or "risk-related aspects" suffered from misclassification. This might, for instance, have happened in cases where authors explicitly labeled their ratings on probability of infection as their measures of risk perception but then also measured severity aspects without further comment on whether this was conceived of as a part of risk perception or not. As most studies provided no theoretical definition or elaboration on how risk perception was conceptualized, it cannot be ruled out that in some cases the apparent distinction in measurement was not a deliberate attempt at conceptual differentiation. Likewise, in some instances where studies were published in rather brief formats, it cannot be excluded that the empirical work actually was theory-based, but, due to extremely short background sections in some articles, this might not have been discernible.

\section{Conclusions}

In a recent study, Wu et al. [73] put forward an epidemiological model which tested pathways to reduce the impact of pandemic influenza by household interventions. The authors reported that already moderate levels of behavioral compliance would bring about substantial population-level benefits and also that individual decisions which would influence compliance might differ widely. The authors concluded that there is a clear need for psychobehavioral surveillance studies to estimate levels of compliance and to identify factors which would influence the decisions of individuals and households.

The presently reviewed body of studies provides preliminary evidence with regard to one such factor, i.e., risk perception, and is valuable insofar as the studies offer first insights into peoples' reaction to new or reemerging health threats such as SARS or avian influenza. However, in order to gather more systematic and compelling data, which can be compared and evaluated within the context of others studies, and in order to build a sound evidence base, future studies in the field positively need to carry out more theoretical groundwork which can guide the development and choice of risk perception concepts and measurements as well as study designs. It was one of the most surprising findings of this review that the risk perception label was often used with little explicit explanation of what it stood for, as if risk perception were a self-evident and homogeneous concept. However, closer scrutiny of the studies revealed that this common label actually covered quite different notions - a, which would make it extremely difficult to draw any generalized conclusions from the resulting body of data. In view of this finding, it might be worth striving for a working consensus on indispensable core measures of risk perception, particularly for future surveillance and monitoring studies. As stated above, many of the studies were initiated in an ad hoc fashion as an acute response to outbreaks of SARS and human H5N1 cases and not as an explicit attempt at testing theory. However, the call for model-based risk perception research [15, 74] does not exclusively aim at benefiting theoretical knowledge about what "is" and what determines risk perception. A thorough understanding of how perceptions of risk develop and in what way and by which mechanisms they influence behavior is an indispensable prerequisite for the development of effective health risk messages and for promoting protective behaviors in case of an outbreak. To define the purpose of a study as predominantly pragmatic in terms of wanting to provide data for planning does not imply nonnecessity for theoretical groundwork. Otherwise, the data gathered might be difficult to interpret and of limited value, not at least for the practical purpose they were intended for in the first place. 
"The threat of an influenza pandemic is, at present, one of the most significant public health issues our nation and world faces" - so a statement of the US Commissioner of Food and Drugs, Andrew von Eschenbach in a press release from April 2007 [75]. While some medical advances have been made, thus for instance a preliminary vaccine has been developed to counteract human-to-human transmission of H5N1, there is still agreement that an initial outbreak cannot be controlled by medical strategies alone. Apart from the fact that the vaccine offers only limited protection, concerns have also been voiced that the medication which has predominantly been stockpiled so far, i.e., Tamiflu (oseltamivir), might be meeting with increasing resistance [76-78]. Also, it is by no means certain that the next epidemic actually will be a $\mathrm{H} 5 \mathrm{~N} 1$ outbreak, or if another new virus will emerge on the scene. Thus, traditional public health containment strategies will still be a centerpiece of preparedness planning. To provide a more profound basis for this type of planning and to enable effective health communication, research on pandemic influenza risk perception will have to progress from its current preliminary ad hoc stage into a more systematic, methodological, and theory-based endeavor.

\section{References}

1. Smith RD. Responding to global infectious disease outbreaks: lessons from SARS on the role of risk perception, communication and management. Soc Sci Med. 2006;63:3113-3.

2. Savage L. The foundations of statistics. New York: Wiley; 1954.

3. Fischhoff B, Slovic P, Lichtenstein S, Read S, Combs B. How safe is safe enough? A psychometric study of attitudes towards technological risks and benefits. Policy Sci. 1978;9:127-52.

4. Slovic P. The perception of risk. London: Earthscan; 2000.

5. Rosenstock IM. Why people use health services. Milbank Mem Fund Q. 1966;44(Suppl):127-36.

6. Becker MH, Rosenstock IM. Comparing social learning theory and the health belief model. In: Ward WB, editor. Advances in health education and promotion. Greenwich: JAI; 1987. p. 245-9.

7. Rogers R. A protection motivation theory of fear appeals and attitude change. J Psychol. 1975;91:93-114.

8. Rogers R. Attitude change and information integration in fear appeals. Psychol Rep. 1985;56:182.

9. Chaiken S. The heuristic model of persuasion. In: Zanna K, Olson J, Herman C, editors. Social influence: the Ontario Symposium. 5th ed. Hillsdale: Erlbaum; 1987. p. 3-39.

10. Petty R, Cacioppo J. The elaboration likelihood model of persuasion. In: Berkowitz L, editor. Advances in experimental social psychology. Burlington: Academic; 1986. p. 123-205.

11. Damasio AR. Descartes' error: emotion, reason, and the human brain. New York: Avon; 1994.

12. Forgas JP. Affect in social judgments and decisions: a multiprocess model. Adv Exp Soc Psychol. 1992;25:227-75.

13. Forgas JP. Mood and judgment: the affect infusion model (AIM). Psychol Bull. 1995;177:39-66.

14. Schwarz N. Feelings as information: informational and motivational functions of affective states. In: Sorrentino RM, Higgins ET, editors. Handbook of motivation and cognition: foundations of social behaviour. New York: Guilford; 1990. p. 527-61.
15. Finucane ML, Holup JL. Psychosocial and cultural factors affecting the perceived risk of genetically modified food: an overview of the literature. Soc Sci Med. 2006;60:1603-12.

16. Finucane ML, Peters E, Slovic P. Judgment and decision making: the dance of affect and reason. In: Schneider SL, Shanteau J, editors. Emerging perspectives on judgment and decision research. Cambridge: Cambridge University Press; 2003. p. 326-64.

17. Loewenstein GF, Weber EU, Hsee CK, Welch N. Risk as feelings. Psychol Bull. 2001;127:267-86.

18. Finucane ML, Alkahani A, Slovic P, Johnson SM. The affect heuristic in judgements of risks and benefits. J Behav Decis Mak. 2000;13:1-17.

19. Slovic P, Finucane ML, Peters E, MacGregor DG. Risk as analysis and risk as feelings: some thoughts about affect, reason, risk, and rationality. Risk Anal. 2004;24:311-22.

20. Lazarus R, Folkman S. Stress, appraisal and coping. New York: Springer; 1984.

21. Roseman IJ, Antoniou AA, Jose PE. Appraisal determinants of emotion: constructing a more accurate and comprehensive theory. Cogn Emot. 1996;10:241-77.

22. Irwin A, Wynne B. Conclusions. In: Irwin A, Wynne B, editors. Misunderstanding science: the public reconstruction of science and technology. Cambridge: Cambridge University Press; 1996. p. 213-21.

23. Moscovici S. Why a theory of social representations. In: Deaux K, Philogene G, editors. Representations of the social. Oxford: Blackwell; 2001. p. 8-35.

24. Kasperson R, Kasperson J. The social amplification and attenuation of risk. Ann Am Acad Polit Soc Sci. 1996;545:95-105.

25. Pidgeon RE, Kasperson RE, Slovic P, editors. The social amplification of risk. Cambridge: Cambridge University Press; 2003.

26. Brewer NT, Weinstein ND, Cuite CL, Herrington JE. Risk perceptions and their relation to risk behavior. Annals Behav Med. 2004;27:125-30.

27. Brewer NT, Chapman GB, Gibbons FX, Gerrard McCaul KD, Weinstein ND. Meta-analysis of the relationship between risk perception and health behavior: the example of vaccination. Health Psychol. 2007;26:136-45.

28. Ajzen I. The theory of planned behavior. Org Behav Human Decis Process. 1991;50:179-211.

29. Fishbein M, Ajzen I. Belief, attitude, intention, and behavior: an introduction to theory and research. Reading: Addison Wesley; 1975.

30. Brown SL. Relationships between risk-taking behaviour and subsequent risk perceptions. Br J Psychol. 2005;96:155-64.

31. Eiser JR, Miles S, Frewer LJ. Trust, perceived risk, and attitudes toward food technologies. J Appl Soc Psychol. 2002;32:2423-33.

32. Cava MA, Fay KE, Beanlands HJ, McCay EA, Wignall R. Risk perception and compliance with quarantine during the SARS outbreak. J Nurs Sch. 2005;37:343-7.

33. Rambaldini G, Wilson K, Rath D, Lin Y, Gold WL, Kapral MK, et al. The impact of severe acute respiratory syndrome on medical house staff: a qualitative study. J Gen Intern Med. 2005;20:381-5.

34. Chong M-Y, Wang W-C, Hsieh W-C, Lee C-Y, Chiu N-M, Yeh W-C, et al. Psychological impact of severe acute respiratory syndrome on health workers in a tertiary hospital. Br J Psychiatry. 2004;185:127-33.

35. Imai T, Takahashi K, Hoshuyama T, Hasegawa N, Lim M-K, Koh D. SARS risk perceptions in healthcare workers, Japan. Emerg Infect Dis. 2005;11:404-10.

36. Tam DKP, Lee S, Lee SS. Impact of SARS on avian influenza preparedness in healthcare workers. Infection 2007;35:320-5.

37. Shiao JS-C, Koh D, Lo L-H, Lim M-K, Guo L. Factors predicting nurses' consideration of leaving their job during the SARS outbreak. Nurs Ethics. 2007;14:5-17.

38. Tang CSK, Wong C-Y. Factors influencing the wearing of facemasks to prevent the severe acute respiratory syndrome among adult Chinese in Hong Kong. Prev Med. 2004;39:1187-93. 
39. Tang CSK, Wong C-Y. Psychosocial factors influencing the practice of preventive behaviors against the severe acute respiratory syndrome among older Chinese in Hong Kong. J Aging Health. 2005;17:490-506.

40. deZwart O, Veldhuijzen IK, Elam G, Aro AR, Abraham T, Bishop $\mathrm{GD}$, et al. Avian influenza risk perception, Europe and Asia. Emerg Infect Dis. 2007;13:290-3.

41. Lau JTF, Yang X, Wong E, Tsui H. Prevalence and factors associated with social avoidance of recovered SARS patients in the Hong Kong general population. Health Educ Res. 2006;21:662-73.

42. Ji L-J, Zhang Z, Usborne E, Guan Y. Optimism across cultures: in response to the SARS outbreak. Asian J Soc Psychol. 2004;7:25-34.

43. Fielding R, Lam WW, Ho EY, Lam H, Hedley AJ, Leung GM. Avian influenza risk perception, Hong Kong. Emerg Infect Dis. 2005; $11: 677-82$

44. Brug J, Aro AR, Oenema A, de Zwart O, Richardus JH, Bishop GD. SARS risk perception, knowledge, precautions, and information sources, the Netherlands. Emerg Infect Dis. 2004;10:1486-9.

45. Leung GM, Ho L-M, Chan SKK, Ho S-Y, Bacon-Shone J, Choy RYL, et al. Longitudinal assessment of community psychobehavioral responses during and after the 2003 outbreak of severe acute respiratory syndrome in Hong Kong. Clin Infect Dis. 2005;40:1713-20.

46. Nickell LA, Crighton EJ, Tracy CS, Al-Enazy H, Bolaji Y, Hanjrah $\mathrm{S}$, et al. Psychosocial effects of SARS on hospital staff: survey of a large tertiary care institution. Can Med Assoc J. 2004;170:793-8.

47. Kristiansen IS, Halvorsen PA, Gyrd-Hansen D. Influenza pandemic: perception of risk and individual precautions in a general population. Cross-sectional study. BMC Public Health. 2007;7:48-54.

48. Lau JTF, Yang X, Tsui HY, Pang E. SARS related preventive and risk behaviours practised by Hong Kong-mainland China cross border travellers during the outbreak of the SARS epidemic in Hong Kong. J Epidemiol Community Health. 2004;58:988-96.

49. Leung GM, Quah S, Ho L-M, Ho S-Y, Hedley AJ, Lee H-P, et al. A tale of two cities: community psychobehavioral surveillance and related impact on outbreak control in Hong Kong and Singapore during the severe acute respiratory syndrome epidemic. Infect Control Hosp Epidemiol. 2004;25:1033-41.

50. Abbate R, Di Giuseppe G, Marinelli P, Angelillo IF. Knowledge, attitudes, and practices of avian influenza, poultry workers, Italy. Emerg Infect Dis. 2006;12:1762-5.

51. Lau JTF, Yang X, Tsui HY, Pang E, Kim JH. SARS preventive and risk behaviours of Hong Kong air travellers. Epidemiol Infect. 2004;132:727-36.

52. Leung GM, Lam T-H, Ho L-M, Ho S-Y, Chan BHY, Wong IOL, et al. The impact of community psychological responses on outbreak control for severe acute respiratory syndrome in Hong Kong. J Epidemiol Community Health. 2003;57:857-63.

53. Hong S, Collins A. Societal responses to familiar versus unfamiliar risk: comparisons of influenza and SARS in Korea. Risk Anal. 2006;26:1247-57.

54. Koh D, Lim MK, Chia SE, Ko SM, Qian F, Ng V, et al. Risk perception and impact of Severe Acute Respiratory Syndrome (SARS) on work and personal lives of healthcare workers in Singapore: What can we learn? Health Med Care Serv Rev. 2005;43:676-82.

55. Koh D, Takahashi K, Lim MK, Imai T, Chia SE, Qian F, et al. SARS risk perception and preventive measures, Singapore and Japan. Emerg Infect Dis. 2005;11:641-2.

56. Lau JTF, Yang X, Tsui H, Kim JH. Monitoring community responses to the SARS epidemic in Hong Kong: from day 10 to day 62. J Epidemiol Community Health. 2003;57:864-70.

57. Chang C-S, Du P-L, Huang I-C. Nurses' perceptions of severe acute respiratory syndrome: relationship between commitment and intention to leave nursing. J Adv Nurs. 2006;54:171-9.

58. Weinstein ND. Optimistic biases about personal risks. Science 1989;8:1232-3.
59. Bandura A. Self-efficacy: the exercise of control. New York: Freeman; 1997

60. Weinstein ND, Sandman PM. A model of the precaution adoption process: evidence form home radon testing. Health Psychol. $1992 ; 11: 170-80$.

61. Smith VK, Johnson FR. How do risk perceptions respond to information: the case of radon. Rev Econ Stat. 1988;70:1-8.

62. Viscusi WK, Evans WN. Estimation of revealed probabilities and utility functions for product safety decisions. Rev Econ Stat. 1998;80:28-33.

63. Tang CSK, Wong C-Y. An outbreak of the severe acute respiratory syndrome: predictors of health behaviours and effect of community prevention measures in Hong Kong, China. Am J Public Health. 2003;93:1887-8.

64. Kobbeltveld T, Brun W, Johnsen BH, Eid J. Risk as feelings or risk and feelings? A cross-lagged panel analysis. J Risk Res. 2005;8:417-37.

65. Borkovec TD, Robinson E, Pruzinsky T, DePree JA. Preliminary exploration of worry, some characteristics and processes. Behav Res Ther. 1983;21:9-16.

66. Morris LW, Davis MA, Hutchings CH. Cognitive and emotional components of anxiety: literature review and a revised worryemotionality scale. J Educ Psychol. 1981;73:541-55.

67. Harris P, Middleton W. The illusion of control and optimism about health: on being less at risk but no more in control than others. $\mathrm{Br}$ J Soc Psychol. 1994;33:3269-386.

68. Siu JY-M. The SARS-associated stigma of SARS victims in the post SARS era of Hong Kong. Qual Health Res. 2008;18:729-38.

69. Yj S. From an epidemic to mass panic: the role of social and cultural elements in infection control of SARS in Hong Kong. Paper presented at the 38th APACPH (Asia-Pacific Academic Consortium for Public Health) Conference 2006 Bangkok, Thailand; 2006.

70. Lai JC, Tao J. Perception of environmental hazards in Hong Kong Chinese. Risk Anal. 2003;23:669-84.

71. Beck U. Risk society: towards a new modernity. London: Sage; 1992.

72. Beck U. World risk society. Cambridge: Polity Press; 1999.

73. Wu JT, Riley S, Fraser C, Leung GM. Reducing the impact of the next influenza pandemic using household-based public health interventions. PLoS Med. 2006;3:e361.

74. Weber EU, Hsee CK. Models and mosaics: investigating crosscultural differences in risk perception and risk preference. Psychon Bull Rev. 1999;6:611-7.

75. FDA/US Food and Drug Administration. FDA approves first U.S. vaccine for humans against the avian influenza virus H5N1. FDA News P07-68 http://www.fda.gov/bbs/topics/NEWS/2007/ NEW01611.html (2007). Accessed June 8, 2008.

76. Eurosurveillance. Eurosurveillance update. http://www.eurosurveillance. org/edition/v13n06/080207 3.asp (2008). Accessed June 8, 2008.

77. Moscona A. Oseltamivir resistance-disabling our influenza defenses. N Engl J Med. 2005;353:2633-6.

78. Moscona A. Medical management of influenza infection. Annu Rev Med. 2008;59:397-413.

79. Singer AC, Nunn MA, Gould EA, Johnson AC. Potential risks associated with the proposed widespread use of Tamiflu. Environ Health Perspect. 2007;115:102-6.

80. Barennes H, Martinez-Aussel B, Vongphrachanh P, Strobel M. Avian influenza risk perceptions, Laos. Emerg Infect Dis. 2007;13:1126-8.

81. Peltz R, Avisar-Shohat G, Bar-Dayan Y. Difference in public emotions, interest, sense of knowledge and compliance between the affected area and the nationwide general population during the first phase of a bird flu outbreak in Israel. J Infect. 2007;55:545-50.

82. Quah SR, Hin-Peng L. Crisis prevention and management during SARS outbreak, Singapore. Emerg Infect Dis. 2004;10: 364-8. 